\title{
Producción y consumo de maíz entre milperos: entre la utopística y la utopía agroecológica
}

\author{
Miguel Ángel Damián Huato ${ }^{1}$ \\ Dora Ma. Sangerman-Jarquín ${ }^{2 \S}$ \\ Artemio Cruz León ${ }^{3}$
}

\begin{abstract}
${ }^{1}$ Instituto de Ciencias-BUAP. Avenida 14 Sur 6301, Ciudad Universitaria, Puebla, México. CP. 72570. (damianhuato@hotmail.com). ${ }^{2}$ Campo Experimental Valle de México-INIFAP. Carretera Los ReyesTexcoco km 13.5, Coatlichán, Texcoco, Estado de México. CP. 56250. Tel. 80008822 22, ext. 85353. (dsangerman.dora@inifap.gob.mx). ${ }^{3}$ Posgrado en Desarrollo Rural Regional-Universidad Autónoma Chapingo. (etnoagronomia1@gmail.com).
\end{abstract}

${ }^{\S}$ Autora para correspondencia: sangerman.dora@inifap.gob.mx.

\section{Resumen}

Se expone una utopística/utopía agroecológica, teorizada desde la epistemología del sur, la agroecología y la transdisciplina. La utopística evalúa la aplicación de innovaciones en el manejo de maíz, identifica y agrupa a los maiceros por sus rendimientos y propone como utopía agroecológica el patrón tecnológico aplicado por los milperos eficientes a nivel local, nutrido por un diálogo de saberes, que pueden ampliar la producción de alimentos de los milperos menos eficientes. En la investigación se halló que todos los productores eficientes manejaron el maíz como milpa, donde interaccionan innovaciones radicales y progresivas y que los maiceros de baja y media eficiencia productiva Cohetzala y San Nicolás no satisfacen plenamente al consumo de maíz, pero si aplicaran el patrón tecnológico de los maiceros eficientes sus rendimientos crecerían en 91$24 \%$ en Cohetzala y en San Nicolás en $150-38 \%$, respectivamente.

Palabras clave: agroecología, epistemología del sur, manejo de milpa, transdisciplina y diálogo de saberes, utopística-utopía.

Recibido: agosto de 2020

Aceptado: octubre de 2020 
México vive una crisis rural sin precedentes; su origen proviene de la aplicación de un modelo de acumulación de capital rapaz y depredador, que tiene como fin resarcir la caída de la tasa de ganancia en el proceso de producción, por la sustitución de capital variable por constante, derivando en un empobrecimiento social y alimentario. A fin con este modelo, se ha promovido la modernización del campo para impulsar la competitividad internacional de algunos bienes agrícolas, eludiendo mejorar la productividad de los maiceros de secano, porque la producción campesina dejó de formar parte de la reproducción del capital global.

Al perder el vínculo con el capital como productor, las políticas públicas los excluyeron de los planes integrales productivos y los incluyeron en proyectos asistenciales para reproducirlos como fuerza de trabajo y no como productores (Rubio, 2000). Esta política pública mermó la producción de maíz nacional y aumentó su importación, originando la pérdida de la autosuficiencia alimentaria a nivel local.

Datos del SIAP (2019), muestran que durante 38 años (1980-2017) el rendimiento nacional promedio de maíz cultivado bajo condiciones de secano aumentó $860 \mathrm{~kg}$, al pasar de 1650 en 1980, a 2510 en 2017, que significó un incremento anual de $1.15 \%$ mermando la autosuficiencia alimentaria, que se alcanza cuando se satisfacen las necesidades alimenticias mediante la producción local (FAO, 2002). Los bajos rendimientos derivaron en mayor importación de maíz amarillo de Estados Unidos de Norteamérica, pasando de 121 mil toneladas en 1992-1993 a casi cuatro millones en 2003-2004, acelerándose a partir de 2008, hasta alcanzar la cifra actual de cerca de 12 millones de toneladas (CEDRSSA, 2017).

Se trata de un maíz de baja calidad nutricional, originando en México cambios significativos en su pauta de consumo de alimentos que se expresó en una mayor obesidad y desnutrición (Brooks, 2012). La exclusión de los milperos también derivó en mayor pluriactividad, lo que transformó a la agricultura en una actividad económica marginal para las familias campesinas obstruyendo, por el lado de producción, las iniciativas y capacidades de los productores y, por el lado del consumo, la ingesta de alimentos básicos (maíz, frijol, calabaza, arvenses) producidos por la unidad familiar.

La producción-consumo de estos granos han sido y son esenciales para satisfacer necesidades auténticas, 'surgidas históricamente y no dirigidas a la mera supervivencia, donde el elemento cultural, moral y costumbre son decisivos y cuya satisfacción es parte constitutiva de la vida normal de los hombres referidos a una determinada clase de una cierta sociedad' (Heller, 1986).

Los bajos o altos rendimientos dependen directamente de la forma en cómo se maneja un cultivo, sea de riego o de secano. Éste, enuncia la forma en cómo el productor combina durante el ciclo agrícola los medios productivos de los que dispone (tierra, trabajo, conocimientos, tecnología), mediante los cuales, ejecuta sucesivamente a nivel de campo varias tareas (barbecho, siembra, labores de cultivo, etcétera), donde puede aplicar tecnologías modernas (híbridos, agroquímicos, etcétera) o campesinas (semillas criollas, asociación de cultivos, etcétera) o un diálogo de saberes, cuando se usan, a la vez, tecnologías modernas (radicales) y campesinas (progresivas). El manejo se encuentra extraordinariamente influido por condiciones de producción endógenas (clima, suelo, flora, fauna, etcétera) y exógenas (programas de fomento agrícola, ingresos familiares, estructura demográfica de la familia, etcétera), las cuales son inalterables a mediano plazo. 
De los factores aplicados en este manejo destaca la tecnología, que representa el conocimiento científico aplicado en la producción, concretada en objetos (máquinas/artefactos) o sistemas de gestión de la actividad económica (Katz, 1999). Innovar significa introducir cambios tecnológicos radicales o cambios incrementales o progresivos (Manual de Oslo, 2006). Los cambios tecnológicos radicales fueron promovidos por la revolución verde acoplada al fordismo que, como modelo de acumulación de capital, articuló a la agricultura al consumo masivo de insumos agrícolas producidos por la industria. La columna vertebral de la revolución verde fue la generación de híbridos y de fórmulas de producción, por razón del método recomendaciones generales, que abarca la experimentación, la validación, la difusión y la adopción de tecnologías.

Las recomendaciones generales han sido reconocidas como el único método científico valido para generar fórmulas de producción, expuestas en los paquetes tecnológicos recomendados por el Instituto Nacional de Investigaciones Forestales, Agrícolas y Pecuarias (INIFAP). Otros conocimientos son considerados como doxa y tropiezo epistemológico que deben ser superados (Castro, 2007). Para De Sousa (2006), se trata de la monocultura del saber y del rigor, reconocida como una sociología de las ausencias: la idea de que el único saber riguroso es el saber científico, $\mathrm{y}$, por tanto, otros conocimientos no tienen la validez ni el rigor científico.

La utopística y utopía agroecológica expuestas en este estudio registra con la primera la violencia epistémica ejercida por la tecnología generada y recomendada por el INIFAP para el manejo de maíz y, con la segunda, estimula la desobediencia epistémica y la de-colonización del conocimiento, al reconocer y revalorar los saberes campesinos aplicados en el manejo de la milpa. La utopística se admite como una evaluación seria de alternativas históricas, como el ejercicio de nuestro juicio respecto a la racionalidad material de otros sistemas históricos alternativos (Wallernstein, 1998). Aquí se asume como una evaluación rigurosa de las innovaciones aplicadas en el manejo de la milpa, para identificar, escalar y potenciar patrones tecnológicos eficientes a nivel local, que son alternativos al paquete tecnológico recomendado por el INIFAP.

Si la utopística cuaja en un patrón tecnológico eficiente, se trocará en utopía agroecológica que como utopía concreta es objetivamente posible, porque su acontecer es científicamente esperable (Bloch, 2004). Este patrón forma parte de la herencia cultural campesina y por ende, involucra conocimientos que están en permanente construcción, en un proceso de devenir, que no es reiteración de lo ya sabido, sino conocimientos planificados de lo que se está haciendo, para que él mismo contribuya a este devenir hacia algo mejor (Bloch, 2004).

Evaluar el manejo de la milpa exige aplicar un enfoque transdisciplinario, que transgrede las fronteras disciplinarias que intervienen en el estudio de un fenómeno. Transgredir significa traspasar varias disciplinas que se complementan e interaccionan entre sí, aunque varias de ellas no estén relacionadas directamente con el manejo de la milpa. Éste fue razonado como totalidad concreta o no sistémica. Así, no se reduce los alcances del estudio a cada práctica agrícola, sello de la unidisciplina, y tampoco sobrevalora la relación independenciadependencia de todo con el todo, típico del enfoque sistémico que segmentaria el estudio del manejo del maíz en subsistemas.

Para estudiar la totalidad concreta hay que considerar la relación que hay entre el manejo de cultivos, con ciertos temas de otras áreas científicas que son pertinentes para explicar las causas teleológicas del manejo. Por ejemplo: la pluriactividad de los productores, un tema propio de la 
economía influye en la especialización que tienen en el manejo de cultivos, lo que se expresará en un manejo eficiente o ineficiente y en mayores o menores rendimientos por hectárea. Pero no es toda la economía la que influye en el manejo, sino únicamente algunas de sus aristas.

Estudiar las innovaciones aplicadas en el manejo de la milpa, exige hacerlo desde el enfoque agroecológico nutrido por un diálogo de saberes, que implica un: a) proceso comunicativo en el cual se ponen en interacción dos lógicas distintas: la del conocimiento científico y la del saber cotidiano, con la intención de comprenderse mutuamente. [...]; b) escenario donde se ponen en juego verdades, conocimientos, sentimientos y racionalidades diferentes, en la búsqueda de consensos, pero respetando los disensos; y c) encuentro entre seres humanos, donde ambos se construyen y fortalecen: un diálogo donde ambos se transforman (Bastidas et al., 2007).

En este trabajo, se considera que el diálogo de saberes debe de concretarse en el manejo de cultivos, mediante la aplicación de tecnologías agrícolas ancestrales (progresivas) y modernas (radicales) inventadas en épocas distintas. Este diálogo es la columna vertebral del enfoque agroecológico, porque es el garante de la mayor productividad, porque de él manan procesos clave, que derivan de las complementariedades, interacciones y sinergias creadas por todos los elementos que se entrecruzan y retroalimentan en el manejo del agroecosistema. Por ello, evaluar las tecnologías aplicadas en este diálogo es esencial para aumentar la producción y el consumo de maíz, satisfactor básico de las necesidades de la familia campesina.

Este trabajo propone una utopía agroecológica, representado por el patrón tecnológico usado por los milperos eficientes, originada de una prolija evaluación de las innovaciones que aplicaron en el manejo de maíz, para aumentar la producción y consumo de alimentos de los milperos menos eficientes. Plantea como hipótesis que: la productividad tiene su origen en el diálogo de saberes que prevalece en el manejo de la milpa, coexisten maiceros con distintos rendimientos y comparten tecnologías y condiciones generales de vida.

\section{La cuestión metodológica}

Para transitar de la utopística a la utopía agroecológica primero se evaluó el manejo de la milpa. Con este fin, se acopió información referida a las condiciones concretas y generales que confluyen en este manejo. Se aplicó una encuesta a una muestra de 60 y 77 productores de Cohetzala y San Nicolás, respectivamente, elegida del padrón del PROCAMPO, ahora PROAGRO productivo. La evaluación implicó conocer las tecnologías radicales y progresivas que aplicaron en dicho manejo, mediante el IATR y el GETP, de forma respectiva.

Para evaluar el IATR que tomó como referente el paquete tecnológico del INIFAP (Cuadro 1). Para ello, se cotejaron las fórmulas de producción expuestas en el paquete, con las prácticas agrícolas que hizo el maicero in situ. Al paquete se le asignó un valor nominal de 100 puntos y se ponderó según el impacto de cada componente en la productividad: 10 fecha de siembra, 20 variedad, 15 densidad de plantas, 25 y 5, para dosis de fertilizantes y fecha de aplicación, 6 y 4 , para tipo y dosis de herbicida, 6 y 4, para tipo y dosis de insecticida y 5 combatir de enfermedades.

Cada valor ponderado se dividió entre dos, donde el primer cociente correspondió al uso de la recomendación y el segundo a su manejo adecuado. Por ejemplo, si un productor usó la dosis de fertilizante del INIFAP se le asignó 25 puntos, si usó otra dosis 12.5 unidades, y si no usó 
fertilizantes su calificación fue cero. Esto se debe a que el INIFAP recomienda fórmulas de producción, probadas experimentalmente durante varios años, y no el uso de cualquier fertilizante.

Cuadro 1. Paquetes tecnológicos recomendado por el INIFAP para el manejo de maíz en Cohetzala y San Nicolás de los Ranchos (SNR), Puebla, México.

\begin{tabular}{|c|c|c|}
\hline Municipio & Práctica/Innovación & Recomendación \\
\hline \multirow{7}{*}{ 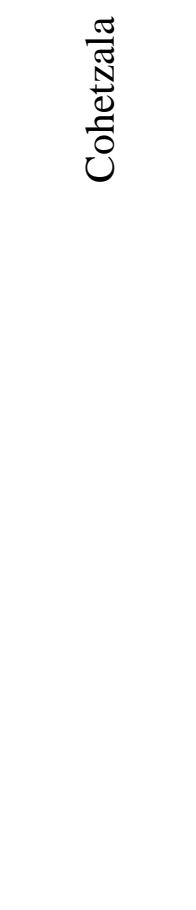 } & Fecha de siembra & Entre marzo-mayo \\
\hline & Tipo de semilla & $\begin{array}{l}\text { H-137, Н-139, Н-34, Н-30, Н-33, Н-40, H-48, H-50, Н-311, Н- } \\
516, \text { H-515, VS-536, Н-507, H-509, V-524, VS-529 y VS-22 }\end{array}$ \\
\hline & $\begin{array}{l}\text { Densidad } \\
\left(\text { plantas } \mathrm{ha}^{-1}\right)\end{array}$ & $50-60$ mil plantas \\
\hline & $\begin{array}{l}\text { Fórmula de } \\
\text { fertilización }\end{array}$ & $120-60-00 ; 100-50-00 ; 180-80-60$ \\
\hline & $\begin{array}{l}\text { Fecha de } \\
\text { fertilización }\end{array}$ & Se aplica en la siembra y segunda labor \\
\hline & $\begin{array}{l}\text { Tipo y dosis } \\
\text { (herbicida } \mathrm{ha}^{-1} \text { ) }\end{array}$ & $\begin{array}{l}\text { Gesaprím } 50(1 \mathrm{~kg}) ; 500 \mathrm{FW}(1.5 \mathrm{~L}) \text {; Gesaprím } 50(1 \mathrm{~kg}) \text { y } \\
\text { Hierbamina }(1 \mathrm{~L}) ;(1 \mathrm{~kg}) \text {; Basagrán } 480(0.5 \mathrm{~L}) \text {; Marvel }(1 \mathrm{~L}) \text {; } \\
\text { Fitoamina } 2.4 \mathrm{D}(1 \mathrm{~L}) \text {, Hierbamina } 2.4 \mathrm{D}(1 \mathrm{~L}) \text {; Esterón } 2.4 \mathrm{D} \\
(1 \mathrm{~L}) \text {. }\end{array}$ \\
\hline & $\begin{array}{l}\text { Tipo y dosis } \\
\text { (insecticida ha } \text { ha }^{-1}\end{array}$ & $\begin{array}{l}\text { Volatón al 2.5\% (25kg); Volatón 5\% (12kg); Furadán 5\% (12 } \\
\text { kg); Folimat } 1000(0.5 \mathrm{~L}) \text {; Parathión metílico 50\% (1 L); } \\
\text { Malathión (1 L); Sevín } 80 \text { (1 kg); Sevín 80\% P H (1 kg); Ma- } \\
\text { lathión } 1000 \text { E (1 L); Diazinón 25\% (1 L). }\end{array}$ \\
\hline \multirow{7}{*}{$\stackrel{\text { 光 }}{\sim}$} & Fecha de siembra & Entre marzo-mayo \\
\hline & Tipo semilla & H-30, H-33, Н-34, H-40, H-48, H-50 H-137, H-139, VS-22 \\
\hline & $\begin{array}{l}\text { Densidad } \\
\left(\text { plantas }^{-1} \mathrm{ha}^{-1}\right)\end{array}$ & 50000 plantas \\
\hline & $\begin{array}{l}\text { Fórmula } \\
\text { fertilización }\end{array}$ & $140-60-00$ y $110-50-00$ \\
\hline & Fecha fertilización & Durante la siembra y segunda labor \\
\hline & 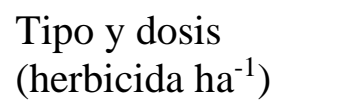 & $\begin{array}{l}\text { Gesaprím } 50(1 \mathrm{~kg}), 500 \text { FW }(1.5 \text { L); Gesaprím } 50 \text { (1 kg) más } \\
\text { Hierbamina }(1 \mathrm{~L}) .\end{array}$ \\
\hline & $\begin{array}{l}\text { Tipo y dosis } \\
\text { (insecticidas } \mathrm{ha}^{-1} \text { ) }\end{array}$ & $\begin{array}{l}\text { Volatón } 2.5 \% \text {, Furadán } 5 \% \text { o Volatón } 5 \% \text { (12-25 kg); Folimat } \\
1000 \text { (0.5 L); Parathión (1 L) metílico 50\% o Malathión (1 L) } \\
\text { disuelto en } 200 \text { L de agua por hectárea. }\end{array}$ \\
\hline
\end{tabular}

$\operatorname{INIFAP}(2009)$.

El cálculo del GETP, correspondió a prácticas agrícolas (asociación y rotación de cultivos, conservación de suelo) e insumos (semilla criolla y estiércol) no idóneos para el INIFAP, pero que los maiceros los aplicaron comúnmente. A cada tecnología se le asignaron 20 unidades. 
Con el cálculo del IATR y del GETP se creó, primero, una tipología de productores agrupándolos según su valor en baja (< a 33.33), media (33.34-66.66) y alta apropiación de tecnología (> a 66.66 unidades), que permitió transitar de las abstracciones empíricas a las constructivas y reflexivas, las cuales fueron esenciales para la construcción del conocimiento (García, 2006). Después se diseñó la utopía agroecológica agrupando a los maiceros por su rendimiento. Para ello, se eligió a los maiceros de mayor y menor rendimiento y la diferencia se tasó entre tres y el cociente se sumó al rendimiento menor para crear tres rangos de tipos de productores: de bajo $(<33.33)$, medio (33.34-66.66) y alto rendimiento por hectárea (> 66.66). Esta tipología permitió analizar el diálogo de saberes implicado en la utopía agroecológica.

Finalmente se estimó el consumo de maíz per capita real y potencial considerando el rendimiento $\left(\mathrm{kg} \mathrm{ha}^{-1}\right)$ real y potencial, el área cosechada y número de miembros de la familia, suponiendo una ingesta de $500 \mathrm{~kg}$ (Warman, 2001). El consumo potencial derivó al considerar que los productores menos eficientes aplicarían el patrón tecnológico de los maiceros eficientes.

\section{La utopística agroecológica}

Al calcular el IATR se halló que: a) el uso de estas innovaciones fue en promedio bajo: 26.7 unidades en Cohetzala y 40.6 en San Nicolás; b) diferenciado, con un rango que va de 22.1-37.3 unidades para productores de Cohetzala de bajo y medio IATR y para San Nicolás de 28.3-44.1, respectivamente, y c) no hubo relación entre el IATR y rendimiento, a pesar de que en Cohetzala hubo 15.2 unidades de diferencia en el IATR entre maiceros de baja (745 kg ha-1) y media (748 $\mathrm{kg} \mathrm{ha}^{-1}$ ). En San Nicolás esta diferencia entre maiceros de baja y media apropiación fue de 15.8 unidades, pero los primeros obtuvieron $1359 \mathrm{~kg} \mathrm{ha}^{-1} \mathrm{y}$ los segundos $1343 \mathrm{~kg} \mathrm{ha}^{-1}$.

La discrepancia que hay entre el uso de innovaciones radicales y rendimientos, se debe a que el manejo convencional lo ha restringido al uso de agroquímicos, que no coincide con las distintas formas de manejo de los maiceros, que lo adecuan a la diversidad de nichos ecológicos locales de México. Además, para los maiceros de secano, la cosecha de maíz es cuestión de sobrevivencia, por lo que sólo han adoptado-adaptado algunas recomendaciones del INIFAP que, al imbricarlos con sus propias tecnologías, deviene en el diálogo de saberes y una mayor productividad.

Por ello, no fue casual que al medir el GETP se halló que: a) en el manejo de maíz prevalecen las innovaciones progresivas sobre las radicales: en Cohetzala el GETP superó en 42.6 unidades al IATR y en San Nicolás, en 20.7; b) el GETP también fue diferenciado: en Cohetzala el rango fluctuó entre 57.5-83 unidades para milperos de medio y alto GETP y en San Nicolás fue entre 2054-82 unidades para maiceros de bajo, medio y alto; y c) hubo una relación directa entre GETP y rendimientos. En Cohetzala estos fueron de $695-816 \mathrm{~kg} \mathrm{ha}^{-1}$, para los de medio y alto GETP, y para San Nicolás de 778-1 285-1 $585 \mathrm{~kg} \mathrm{ha}^{-1}$, expuestos en el mismo orden.

\section{De la utopística a la utopía agroecológica}

La utopística muestra la relevancia de las tecnologías progresivas en el manejo de maíz, pero lo esencial fue que permitió construir la utopía agroecológica. Aplicando la metodología propuesta se halló: los rendimientos $\left(\mathrm{kg} \mathrm{ha}^{-1}\right)$ mayor y menor de los maiceros de Cohetzala fue de $400 \mathrm{y}$ 1000 y los de San Nicolás de 500 y 2 200, la diferencia fue de 600 para Cohetzala y 1700 para 
San Nicolás; el cociente fue de 200 y 567 para Cohetzala y San Nicolás; y rangos, afines a los tres tipos de maiceros según su rendimiento fueron, para Cohetzala: bajos <600, medios entre 601-800 y altos >800 kg ha-1 y para los de San Nicolás fueron: bajos <1 067, medios 1 068-1 635 y altos $>1635$.

Los atributos de los tipos de productores se registraron en el Cuadro 2, donde se nota que: a) casi una tercera parte fueron eficientes, con rendimientos significativamente mayores que los no eficientes; y b) los valores del IATR y del GETP se redujeron debido, tal vez, al impacto que tienen ambas innovaciones en el impulso de las fuerzas productivas, cuando actúan asociadas.

Cuadro 2. Número de productores, IATR, GETP y rendimientos $\left(\mathrm{kg} \mathrm{ha}^{-1}\right)$, según su rendimiento por hectárea de Cohetzala y San Nicolás de los Ranchos (SNR), Puebla, México.

\begin{tabular}{|c|c|c|c|c|c|c|c|c|c|}
\hline \multirow{2}{*}{\multicolumn{2}{|c|}{ Municipios/indicador }} & \multicolumn{2}{|c|}{ Baja } & \multicolumn{2}{|c|}{ Media } & \multicolumn{2}{|c|}{ Alta } & \multicolumn{2}{|c|}{ Promedio municipal } \\
\hline & & Núm. & $(\%)$ & Núm. & $(\%)$ & Núm. & $(\%)$ & Núm. & $(\%)$ \\
\hline \multirow{4}{*}{ 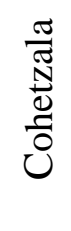 } & Productores & 14 & 23 & 27 & 45 & 19 & 32 & 60 & 100 \\
\hline & IATR & 23.4 & & 27.3 & & 28.3 & & 26.7 & \\
\hline & GETP & 58.6 & & 73.3 & & 71.6 & & 69.3 & \\
\hline & Rendimiento & $486^{\mathrm{a}}$ & & $751^{\mathrm{b}}$ & & $930^{\mathrm{c}}$ & & 746 & \\
\hline \multirow{4}{*}{ 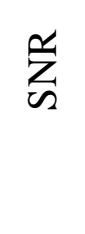 } & Productores & 27 & 35 & 28 & 36 & 22 & 29 & 77 & 100 \\
\hline & IATR & 44.1 & & 35.8 & & 42.3 & & 40.6 & \\
\hline & GETP & 48.1 & & 64.3 & & 73.6 & & 61.3 & \\
\hline & Rendimiento & $763^{a}$ & & $1438^{b}$ & & $1971^{\mathrm{c}}$ & & 1347 & \\
\hline
\end{tabular}

Elaborado con datos obtenidos de la encuesta, 2009. Dentro de cada hilera (rendimiento), letras distintas en las medias indica que hay diferencia estadística significativa entre ellas (prueba de Tukey, $p<0.05$ ).

Al comparar los patrones tecnológicos aplicados por los maiceros (Cuadro 3) con los del INIFAP, se advierte que los segundos promueven la violencia epistémica en el manejo de la milpa ya que el instituto no reconoció las tecnologías campesinas, decisivos en este manejo, en tanto que los primeros impulsaron la independencia epistémica y la de-colonización del conocimiento, al reconocer y valorar los saberes campesinos aplicados en el manejo de la milpa. Asimismo, se observa que en los primeros se aplicó un diálogo de saberes, máxime los productores eficientes, de donde derivó su mayor productividad, habilitada por cuatro procesos tecnológicos que desglosan con fines analíticos, pero que ocurren a la par.

El primero es alentado por la biomimesis propia de la asociación de cultivos la cual consta de varios 'pisos de plantas', como en los ecosistemas naturales, originando mayor diversidad de flora y fauna arriba-abajo del suelo y adentro-alrededor del agroecosistema. En esta diversidad, coexisten plantas C4 (maíz) y C3 (calabaza, frijol, etc.) con necesidades distintas de energía solar para convertir compuestos inorgánicos en orgánicos, eficientando el uso de energía solar. También la diversidad de flora y fauna adentro-alrededor de la milpa impulsa la polinización y crea redes tróficas que reducen el daño de patógenos a la milpa. 
Cuadro 3. Innovaciones aplicadas en manejo de la milpa, respecto al área total sembrada (ha), según la productividad de los milperos de Cohetzala y San Nicolás de los Ranchos, Puebla, México.

\begin{tabular}{|c|c|c|c|c|c|}
\hline Municipio & Dato/innovación & Baja & Media & Alta & Promedio municipal \\
\hline \multirow{11}{*}{$\begin{array}{l}\frac{\pi}{\pi} \\
\frac{\mathbb{N}}{0} \\
\frac{0}{0}\end{array}$} & Área sembrada maíz (ha) & 29.5 & 61 & 47 & 137.5 \\
\hline & Conservación suelos (\%) & 14 & 36 & 23 & 27 \\
\hline & Semilla criolla $(\%)$ & 100 & 100 & 100 & 98 \\
\hline & Asociación de cultivos (\%) & 93 & 100 & 100 & 99 \\
\hline & Asociación con leguminosas (\%) & 83 & 66 & 100 & 81 \\
\hline & Asociación con calabaza (\%) & 63 & 66 & 98 & 76 \\
\hline & Rotación de cultivos (\%) & 27 & 46 & 45 & 41 \\
\hline & Aplicación estiércol $\left(\mathrm{kg} \mathrm{ha}^{-1}\right)$ & 1434 & 1677 & 1775 & 1644 \\
\hline & Aplicación fertilizante (\%) & 83 & 93 & 100 & 93 \\
\hline & Aplicación herbicida (\%) & 29 & 18 & 15 & 19 \\
\hline & Aplicación insecticida (\%) & 0 & 9 & 19 & 11 \\
\hline \multirow{11}{*}{ 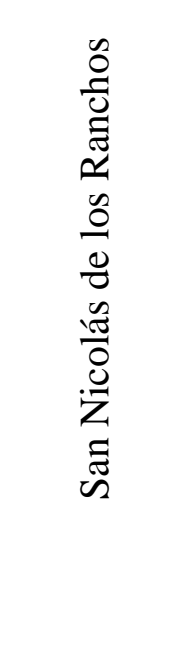 } & Área sembrada maíz (ha) & 62.25 & 77 & 79.5 & 218.75 \\
\hline & Conservación suelos (\%) & 81 & 69 & 61 & 70 \\
\hline & Semilla criolla $(\%)$ & 97 & 100 & 100 & 99 \\
\hline & Asociación de cultivos (\%) & 31 & 34 & 31 & 32 \\
\hline & Asociación con leguminosas (\%) & 3 & 32 & 26 & 30 \\
\hline & Asociación con calabaza (\%) & 0 & 0 & 3 & 1 \\
\hline & Rotación de cultivos (\%) & 48 & 64 & 84 & 67 \\
\hline & Aplicación estiércol $\left(\mathrm{kg} \mathrm{ha}^{-1}\right)$ & 550 & 590 & 1383 & 803 \\
\hline & Aplicación fertilizante (\%) & 96 & 79 & 96 & 90 \\
\hline & Aplicación herbicida (\%) & 85 & 36 & 52 & 56 \\
\hline & Aplicación insecticida (\%) & 43 & 14 & 25 & 26 \\
\hline
\end{tabular}

Elaborado con datos obtenidos de la encuesta, 2009.

A la par, esta diversidad provee más biomasa abajo del suelo, redundando en mayor abundancia y riqueza de microorganismos que descomponen la materia orgánica y reciclan los nutrientes y la energía. A la vez, en un policultivo la gama de colores y olores liberados por la vegetación dificulta la ubicación del alimento por los insectos (Paleologos y Flores, 2014). El bajo uso de insecticidas por los milperos puede ser resultado de esta diversidad de especies, ya que crea un hábitat idóneo para que haya mayor abundancia y riqueza de artropodofauna, derivando en un equilibrio biológico del sistema milpa. El paquete tecnológico del INIFAP considera a elementos de la artropodofauna como plagas, proponiendo su exterminio, perturbando este equilibrio biológico.

Walker (1992) reporta que si en esta biodiversidad hay redundancia de especies por el rol que juegan en el agroecosistema, se creará una redundancia de relaciones que asegura pocos cambios en los agroecosistemas (estabilidad) y de ocurrir un cambio ambiental extremo, el agroecosistema 
tiene mayor capacidad para absorber las perturbaciones y para recuperar pronto su funcionalidad (resiliencia). Un sistema resiliente subsiste más en el tiempo, o sea, es sostenible. Las especies redundantes están representadas por las arvenses, calificadas por la agricultura moderna como malezas, planteado su aniquilamiento y con ello la redundancia de relaciones que promueven.

El segundo proceso agroecológico deriva de las plantas asociadas, potenciando la relación aguasuelo-planta-ambiente: el frijol fija nitrógeno atmosférico, nutriente esencial de las plantas, el maíz le sirve de tutor al frijol, y la calabaza, con su amplio follaje y hábito rastrero, salvaguarda al suelo de la erosión, fomenta la filtración de agua e impide su evaporación y el crecimiento de arvenses durante los primeros ciclos fenológicos del maíz y frijol.

El tercer proceso mana de las innovaciones campesinas. La adaptación milenaria de las semillas criollas a los nichos ecológicos locales y su estabilidad productiva se debe a su alta variabilidad, que está en permanente cambio (Flores y Sarandón 2014). La rotación de cultivos mejora las propiedades de los suelos, retiene humedad y nutrientes, aportan nutrientes y sanidad al suelo (Mendoza, 2004). La conservación de suelos evita la pérdida de nutrientes y agua, los cuales potencian su productividad. El estiércol provee nutrientes, mejora la estructura y textura, aumenta la aireación, la penetración y retención de agua, estimula el desarrollo de microorganismos benéficos para la planta y promueve la captura de carbono (Robert, 2002).

Finalmente, un proceso deriva de la fusión de las tecnologías campesinas y algunas modernas, como la aplicación de fertilizantes nitrogenados. El nitrógeno es esencial para el crecimiento de las plantas, además, es primordial para el crecimiento microbiano y la degradación de la materia orgánica. Si ésta tiene alto contenido de nitrógeno, los microorganismos tendrán suficiente sustrato para inducir mayor mineralización, pues la microflora tendrá plenamente satisfechas sus necesidades de nitrógeno (Ferrera y Alarcón, 2001). Destaca que en Cohetzala y San Nicolás, 78.3 y $68.8 \%$ de los milperos aplicaron sólo fertilizantes nitrogenados.

Datos del Cuadro 4 muestran que los tipos de milperos tienen condiciones de vida similares: son minifundistas extremos y con escasos medios de producción; sus gastos promedios son muy bajos y están fuertemente subsidiados por las remesas, máxime en Cohetzala, la producción se destina al autoconsumo y despliegan varias estrategias de sobrevivencia: la venta de fuerza de trabajo, el manejo de la milpa, del ganado mayor y menor, del traspatio y la recolección de bienes naturales. No obstante, los eficientes se caracterizaron porque diversificaron sus tareas en el sector primario relacionados con el manejo de la milpa, sobre todo en Cohetzala, mientras que los demás, lo han hecho en los sectores secundario y terciario.

La pluriactividad aumenta el ingreso de los hogares rurales (De Grammont, 2009) y a la vez, reduce la renta agrícola (Anseeuw y Laurent, 2007). La pluriactividad ha convertido a la agricultura en una tarea económica marginal para la unidad familiar, obstruyendo las iniciativas y capacidades de los productores que nutren el diálogo de saberes y con ello, los procesos tecnológicos clave que acaecen en la milpa.

Por último, en los datos del Cuadro 4 destaca el alto porcentaje de maíz destinado al autoconsumo, lo que sugiere que este bien posee el más alto aprovechamiento social derivado de la actividad humana, debido a que se destina directamente a la satisfacción de las necesidades humanas. 
Cuadro 4. Rasgos socioeconómicos y disponibilidad a medios de producción, según el rendimiento de los productores de Cohetzala y San Nicolás de los Ranchos, Puebla, México.

\begin{tabular}{|c|c|c|c|c|c|}
\hline & Municipios/Indicadores & Baja & Media & Alta & Promedio municipal \\
\hline \multirow{16}{*}{$\begin{array}{l}\frac{\pi}{\pi} \\
\frac{\mathbb{N}}{0} \\
\frac{\pi}{0}\end{array}$} & Edad & 54.8 & 57.8 & 52.6 & 55.5 \\
\hline & Migrantes/familia (promedio) & 2 & 3 & 2.4 & 2.6 \\
\hline & Remesas (\$promedio/mes/per capita) & 475 & 607 & 474 & 534 \\
\hline & Gasto prom. (\$/mes/per capita) & 927 & 1132 & 1001 & 1043 \\
\hline & Autoconsumo de maíz (\%) & 100 & 89 & 68 & 83 \\
\hline & Autoconsumo y venta de maíz (\%) & 0 & 11 & 32 & 17 \\
\hline & Pluriactivos primarios $^{*}(\%)$ & 43 & 67 & 95 & 70 \\
\hline & Pluriactivos secundarios ${ }^{* *}(\%)$ & 29 & 15 & 5 & 15 \\
\hline & Maicero $^{* * *}(\%)$ & 28 & 18 & 0 & 15 \\
\hline & Área sembrada maíz (promedio ha) & 2.1 & 2.3 & 2.5 & 2.3 \\
\hline & Área de traspatio $\left(\mathrm{m}^{2}\right.$ promedio $)$ & 409 & 515 & 317 & 427 \\
\hline & Recolección de bienes (\%) & 100 & 89 & 89 & 92 \\
\hline & Posesión de tractor (\%) & 14 & 15 & 5 & 7 \\
\hline & Posesión de yunta (\%) & 71 & 70 & 84 & 75 \\
\hline & Ganado mayor (núm. cabezas/promedio) & 4.5 & 6.1 & 5.8 & 5.6 \\
\hline & Ganado menor (núm. cabezas/promedio) & 10.6 & 18.2 & 19.3 & 16.8 \\
\hline \multirow{16}{*}{ 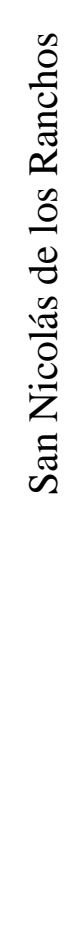 } & Edad & 58.5 & 52.4 & 52.1 & 54.5 \\
\hline & Migrantes/familia (prom.) & 1.1 & 1.1 & 1 & 1.1 \\
\hline & Remesas (\$/mes/per capita) & 112 & 94 & 91 & 100 \\
\hline & Gasto prom. (\$/mes/per capita) & 726 & 657 & 648 & 677 \\
\hline & Autoconsumo de maíz (\%) & 78 & 36 & 9 & 43 \\
\hline & Autoconsumo y venta de maíz (\%) & 22 & 64 & 91 & 57 \\
\hline & Pluriactivos primarios $^{*}(\%)$ & 15 & 64 & 100 & 57 \\
\hline & Pluriactivos secundarios ${ }^{* *}(\%)$ & 70 & 25 & 0 & 34 \\
\hline & Maicero $^{* * *}(\%)$ & 15 & 11 & 0 & 9 \\
\hline & Área sembrada maíz (promedio ha) & 2.3 & 2.8 & 3.6 & 2.8 \\
\hline & Área de traspatio $\left(\mathrm{m}^{2}\right.$ promedio) & 191 & 197 & 356 & 240 \\
\hline & Recolección de bienes (\%) & 96 & 93 & 100 & 95 \\
\hline & Posesión de tractor (\%) & 7 & 7 & 18 & 10 \\
\hline & Posesión de yunta (\%) & 43 & 54 & 77 & 57 \\
\hline & Ganado mayor (núm. cabezas/promedio) & 1.2 & 2.2 & 6.9 & 3.2 \\
\hline & Ganado menor (núm. cabezas/promedio) & 9.8 & 12.4 & 23.3 & 14.6 \\
\hline
\end{tabular}

Elaborado con datos de la encuesta, 2009. ${ }^{*}=$ productores que cultivaron maíz y realizaron otras tareas en ramas económicas del sector primario; ${ }^{* *}=$ ejecutaron otras actividades en los sectores secundario y terciario; ${ }^{* * *}=$ incluye a productores que sólo sembraron maíz. 


\section{Producción y consumo de maíz}

Datos de la encuesta indican que la estructura familiar de los maiceros fue de 328 y 401 personas para Cohetzala y San Nicolás respectivamente. Al estimar el consumo de maíz real se halló que: a) 15 y $57 \%$ de las personas de Cohetzala y San Nicolás, lograron la autosuficiencia alimentaria al producir, al menos, $500 \mathrm{~kg}$ per capita. Si se transfiere el patrón tecnológico de los maiceros eficientes de Cohetzala a los de baja y media eficiencia, los rendimientos crecerían, en promedio, 91 y 24\%; por ello, 27\% de personas alcanzarían la autosuficiencia alimentaria en Cohetzala. Para San Nicolás el rendimiento aumentaría 150 y 38\%, para maiceros de baja y media eficiencia productiva, permitiendo que $81 \%$ de personas consigan la autosuficiencia alimentaria.

Estos resultados coinciden con los de otros autores. Pretty et al. (2011), al evaluar 40 proyectos agrícolas de 20 países africanos durante 2001-2010 donde se aplicó la lucha integrada contra plagas, la conservación de suelos y la agroforestería. En 2010, el rendimiento medio se multiplicó por 2.13 e incrementó la producción total de alimentos en 5.8 millones de toneladas al año, equivalente a $557 \mathrm{~kg}$ por familia/año. Por su parte, el Movimiento Campesino a Campesino (Holt, 2008) aumentó los rendimientos del maíz de media tonelada por hectárea a tres, al aplicar composta y rotaciones con leguminosas, así como de intercalar nuevos cultivos en las parcelas de Vicente Guerrero del municipio de Españita-Tlaxcala-México.

\section{Conclusiones}

Los resultados obtenidos confirman las hipótesis planteadas. En efecto, encontramos que: coexisten maiceros con distintos niveles de apropiación de tecnologías radicales y progresivas, y rendimientos diferenciados; la productividad tiene su origen en el diálogo de saberes, donde el manejo de la biodiversidad presente en la milpa ha sido y será, fundamental para optimizar los rendimientos unitarios, finalmente se halló, que los maiceros comparten tecnologías ya que la gran mayoría aplicaron tecnologías modernas y campesinas, con una evidente prevalencia de las segundas; además, al poseer condiciones generales de vida similares, facilitaría a los maiceros menos eficientes apropiarse del patrón tecnológico que aplicaron los milperos eficientes, asumida como utopía agroecológica. Ésta, como se demostró, puede potenciar el desarrollo de las fuerzas productivas que dormitan en el seno de la tierra y del trabajo, porque está preñada de lo objetivamente posible y se encuentran disponibles entre los productores a nivel local. En la utopía agroecológica, prevalece un trabajo útil, socialmente necesario, que produce bienes auténticos, destinados al autoconsumo de las familias campesinas. Este ha sido el rol que ha desempeñado la milpa desde tiempos inmemoriales.

\section{Literatura citada}

Anseeuw, W. and Laurent, C. 2007. Occupational paths towards commercial agriculture: the key roles of farm. J. Arid Environ. 70(4):659-671.

Bastidas, M.; Pérez, Torres, F.; Escobar, J.; Arango, G. and Peñaranda, A. F. 2009. El diálogo de saberes como posición humana frente al otro: referente ontológico y pedagógico en la educación para la salud. Universidad de Antoquia. 27(1):104-111.

Bloch, E. 2004. Principio esperanza-I. (Ed.). Trotta. Madrid, España. 515 p.

Brooks, D. 2012. Obesidad, regalo del TLCAN a México, periódico La Jornada, 06 de abril. 36 p. 
Castro, S. 2007. Decolonizar la universidad: la hybris del punto cero y el diálogo de saberes. In: Castro, S. y Grosfoguel, R. (Ed.). El giro decolonial: reflexiones para una diversidad epistémica más allá del capitalismo global. Siglo del hombre, Colombia. 79-92 pp.

CEDRSSA. 2017. Centro de Estudios para el Desarrollo Rural Sustentable y la Soberanía Alimentaria. Reporte. El Tratado de Libre Comercio de América de Norte, el sector agroalimentario mexicano y la llegada de Donald Trump a la presidencia de los Estados Unidos. Palacio Legislativo de San Lázaro, Ciudad de México. 21 p.

De Grammont, H. 2009. La nueva estructura ocupacional en los hogares rurales mexicanos. La pluriactividad en el campo mexicano. Ecuador. FLACSO. 273-303 pp.

De Sousa, S. 2006 Renovar la teoría crítica y reinventar la emancipación social. Encuentros en Buenos Aires. CLACSO, Buenos Aires. ISBN: 10:987-1183-57-7. 110 p.

FAO. 2002. Organización de las Naciones Unidas para la Alimentación y la Agricultura. Agua y cultivos. Logrando el uso óptimo del agua en la agricultura. http://www.fao.org/docrep/ 005/Y3918S/ Y3918S00.HTM.

Ferrera, R. y Alarcón, A. 2001. La microbiología del suelo en la agricultura sostenible Ciencia Ergo Sum. Universidad Autónoma del Estado de México (UAEM). México. 8(2):175-183.

Flores, C. y Sarandón, S. 2014. Desarrollo y evolución de los ecosistemas. In: Sarandón, S. y Flores, C. (Ed.). Agroecología: bases teóricas para el diseño y manejo de Agroecosistemas sustentables. Universidad Nacional de La Plata. Argentina. 466 p.

García, R. 2006. Sistemas complejos. Conceptos, método y fundamentación epistemológica de la investigación interdisciplinaria. Gedisa, España. 201 p.

García, A. 2009. Forma valor y forma comunidad. Aproximación teórica-abstracta a los fundamentos civilizatorios que preceden al Ayllu Universal. Muela del Diablo Editores, CLACSO. La Paz Bolivia. 367 p.

Heller, A. 1986. Teoría de las necesidades en Marx. Ediciones Península. 2a (Ed.). Barcelona, España. 182 p.

Holt, G. 2008. Campesino a campesino. Managua Nicaragua. Voces de Latinoamérica Movimiento Campesino para la Agricultura Sustentable. 294 p.

INIFAP. 2009. Instituto Nacional de Investigaciones Forestales, Agrícolas y Pecuarias. Base de datos CD-ROM. Paquete tecnológico para el cultivo de maíz, Distrito de Desarrollo Rural de Cholula e Izúcar de Matamoros, Puebla-México.

Katz, C. 1999. La tecnología como fuerza productiva social: Implicancias de una caracterización. Rev. Latinoam. Historia Cienc. Tecnol. 12(3):371-381.

López, M. A. y Alvarado, J. 2010. Interpretación de nomogramas de análisis de vectores para diagnóstico nutrimental de especies forestales. Madera y Bosques. 16(1):99-108.

Manual de Oslo. 2006. Guía para la recogida e interpretación de datos sobre innovación. Tercera edición. Organización de Cooperación y Desarrollo Económico y Oficina de Estadísticas de las Comunidades Europeas, España. 188 p.

Mendoza, R. 2004. Otras prácticas de cultivo de los productores de maíz: diversificación, rotación de cultivos y técnicas de conservación de suelos, pp. 197-198. In: Damián, M. A.; Ramírez, B.; Gil, A.; Gutiérrez, N.; Aragón, A.; Mendoza, R.; Paredes, J. C.; Damián T. y Almazán. Apropiación de tecnología agrícola. Características técnicas y sociales de los productores de maíz de Tlaxcala. Puebla: BUAP-CONACYT-SIZA y H. Congreso del estado de Tlaxcala, Puebla, México, 191-207 pp.

Paleologos, M. y Flores, C. 2014. Principios para el manejo ecológico de plagas. In: Sarandón, S. y Flores, C. (Ed.). Agroecología: bases teóricas para el diseño y manejo de aes sustentables. Argentina. Facultad de Ciencias Agrarias y Forestales. 260-285 pp. 
Pretty, J. Toulmin, C. and Williams, S. 2011. Sustainable intensification in African Agriculture. Inter. J. Agric. Sustainab. 9(1):5-24.

Robert, M. 2002. Captura de carbono en los suelos para un mejor manejo de la Tierra. Informes sobre recursos mundiales de suelos, Organización de las Naciones Unidas para la Agricultura y la Alimentación. Roma, Italia. 5 p.

Rubio, B. 2000. Los campesinos latinoamericanos frente al nuevo milenio. Rev. Comercio Exterior. 50(3):269-271.

SIAP. 2017. Servicio de Información Agroalimentaria y Pesquera. Series históricas de superficie sembrada y cosechada, 1980-2014, SAGARPA. http://www.siap.gob.mx/index.php? option=com - wrapper $\&$ view $=$ wrapper $\&$ Itemid $=351$.

Walker, B. 1992. Biodiversity and ecological redundancy. Conservation Biology. (6):18-23.

Wallernstein, I. 1998. Utopística o las opciones históricas del siglo XXI. Siglo Veintiuno Editores, México. $91 \mathrm{p}$.

Warman, A. 2001. El campo mexicano en el siglo XX. Fondo de Cultura Económica (FCE). México, DF. 262 p. 